\title{
The $g$ factor of an electron or muon bound by an arbitrary central potential
}

\author{
S.G. Karshenboim ${ }^{(a, b) *}$, R.N. Lee ${ }^{(c) \dagger}$, A. I. Milstein ${ }^{(c) \ddagger}$ \\ ${ }^{(a)}$ D. I. Mendeleev Institute for Metrology (VNIIM), St. Petersburg 198005, Russia \\ (b) Max-Planck-Institut für Quantenoptik, 85748 Garching, Germany \\ (c) Budker Institute of Nuclear Physics, 630090 Novosibirsk, Russia.
}

(Dated: July 3, 2018)

\begin{abstract}
We consider the $g$ factor of a spin-1/2 particle (electron or muon) bound by an arbitrary central field. We present an approach which allows one to express the relativistic $g$ factor in terms of the binding energy. We derive the general expression for the correction to the $g$ factor caused by a deviation of the central potential from the Coulomb one. As the application of this method, we consider the corrections to the $g$ factor due to the finite nuclear size, including vacuum polarization radiative correction. The effect of the anomalous magnetic moment is also taken into account.
\end{abstract}

PACS numbers: 24.80.+y, 25.30.Bf, 21.10.Gv

\section{INTRODUCTION}

Study of energy levels of atomic electron (muon) has a long history. Various relativistic and radiative corrections have been obtained in analytic or semi-analytic form taking into account a deviation of the potential from the Coulomb one. Recently interest to an accurate theory of the $g$ factor increased. Accurate theoretical approach needs to take into account relativistic and radiative corrections. Here we consider a case when such corrections correspond to some modification of a central potential. Such a problem is actual because of the Uehling potential, which is responsible for a dominant QED correction in muonic atoms, finite-nuclear-size effects and some others. The results are presented in a fully relativistic approach, i.e. exact in $Z \alpha$ for the Coulomb interaction and its modifications.

The methods of calculation of energy levels have been successfully developed over the decades. The energy levels can be calculated with high accuracy by solving certain equations analytically or numerically, or using the perturbation theory with respect to the corresponding correction to the hamiltonian. Calculation of the $g$ factor is a more complicated problem. In the traditional approach to the calculation of the correction to the $g$ factor due to modification of the potential $\delta V$, one considers both the magnetic field and $\delta V$ as a perturbation, thus starting with the second order of the perturbation theory. The numerical approaches are also not easy to apply because they usually are much more accurate in determination of the energy than the wave function. However, it is the latter which is needed to calculate the $g$ factor.

In this paper, we develop a framework which allows one to express the $g$ factor of a Dirac particle bound in arbitrary central potential via the binding energy. For a small deviation of this potential from the Coulomb one,

\footnotetext{
*Electronic address: sek@mpq.mpg.de

$\dagger$ Electronic address: R.N.Lee@inp.nsk.su

${ }^{\ddagger}$ Electronic address: A.I.Milstein@inp.nsk.su
}

we derive the general expression for the first correction to the $g$ factor due to this deviation. The results are valid both for muonic and electronic atoms.

\section{GENERAL RELATIONS}

In the weak homogenous magnetic field $\boldsymbol{B}$, the correction to energy levels of a Dirac particle bound by a central potential reads

$$
\begin{aligned}
\Delta E & =e \int d^{3} r \bar{\Psi}(\boldsymbol{r})(\boldsymbol{\gamma} \cdot \mathbf{A}) \Psi(\boldsymbol{r}) \\
& =\frac{e \boldsymbol{B} \cdot\langle\boldsymbol{J}\rangle}{2 m} g,
\end{aligned}
$$

where

$$
\mathbf{A}=\frac{1}{2}[\boldsymbol{B} \times \mathbf{r}]
$$

The relativistic units in which $\hbar=c=1$ are applied throughout the paper; the charge of the bound particle is $-e, e$ is a charge of a proton, $e^{2}=\alpha=1 / 137$ is the fine structure constant.

Writing the Dirac wave function $\psi(\boldsymbol{r})$ in the form

$$
\psi(\boldsymbol{r})=\left(\begin{array}{c}
f_{1}(r) \Omega \\
i f_{2}(r) \widetilde{\Omega}
\end{array}\right)
$$

where $\Omega$ is the spherical spinor [1] with the angular momentum $J$ and orbital momentum $L, \widetilde{\Omega}=-(\boldsymbol{\sigma} \cdot \boldsymbol{n}) \Omega$, we obtain for the $g$ factor of a bound Dirac particle

$$
g=\frac{2 m \kappa}{j(j+1)} \int d r r^{3} f_{1}(r) f_{2}(r),
$$

$\kappa=j+1 / 2$ for $l>j$ and $\kappa=-(j+1 / 2)$ for $l<j$. The integral in the right-hand side of Eq. (4) can be presented as follows (see, e.g., [2])

$$
\int d r r^{3} f_{1} f_{2}=-\frac{1}{4 m}\left[1-2 \kappa \int d r r^{2}\left(f_{1}^{2}-f_{2}^{2}\right)\right] \text {. }
$$


This formula is valid for the Dirac equation with any central potential. Eq. (4) has been known for a while (see, e.g., 2]), but, to the best of our knowledge, it was never applied to a non-Coulomb problems.

Using the identity

$$
\int d r r^{2}\left(f_{1}^{2}-f_{2}^{2}\right)=\left\langle\gamma_{0}\right\rangle
$$

we have

$$
g=-\frac{\kappa\left[1-2 \kappa\left\langle\gamma_{0}\right\rangle\right]}{2 j(j+1)} .
$$

Assuming that the potential $V(r)$ is independent of the mass of the bound particle, we find

$$
\left\langle\gamma_{0}\right\rangle=\left\langle\frac{\partial H}{\partial m}\right\rangle=\frac{\partial E}{\partial m},
$$

where $H$ is the Dirac hamiltonian $H=\gamma_{0}(\boldsymbol{\gamma} \cdot \boldsymbol{p})+\gamma_{0} m+$ $V(r)$.

Thus we arrive at an equation, which expresses the $g$ factor of the state via its binding energy

$$
g=-\frac{\kappa}{2 j(j+1)}\left[1-2 \kappa \frac{\partial E}{\partial m}\right] .
$$

This equation can be used even in the case when a potential substantially differs from the Coulomb one, as for heavy muonic atoms. In many cases, Eq. (9) essentially simplifies calculation of the corrections to the $g$ factor of a Dirac particle. In particular, it can be applied to such problems as finite-nuclear-size effect and vacuum polarization.

The equation (9) becomes essentially simpler if the potential is close to the Coulomb one, and the deviation $\delta V(r)=V(r)-V_{C}(r)$ can be treated as a perturbation. For the pure Coulomb case, when $\partial E_{\mathrm{C}} / \partial m=E_{\mathrm{C}} / m$, we immediately obtain the well-known result (see, e.g., [3, 4])

$$
\Delta g_{\mathrm{C}}=-\frac{\kappa}{2 j(j+1)}\left[1-2 \kappa \frac{\gamma+n_{r}}{N}\right] .
$$

where

$$
N=\sqrt{\left(\gamma+n_{r}\right)^{2}+(Z \alpha)^{2}}, \quad \gamma=\sqrt{\kappa^{2}-(Z \alpha)^{2}},
$$

and $n_{r}$ is the radial quantum number. The correction to the energy is

$$
\delta E=\int d^{3} r \Psi^{+}(r) \delta V(r) \Psi(r) .
$$

As follows from the dimensional reasons, the wave function in the Coulomb field can be presented in the form $\Psi(r)=m^{3 / 2} \widetilde{\Psi}(m r)$, where $\widetilde{\Psi}$ is dimensionless. Passing to the variable $\rho=m r$, we find

$$
\delta E=\int d^{3} \rho \widetilde{\Psi}^{+}(\rho) \delta V(\rho / m) \widetilde{\Psi}(\rho) .
$$

Taking the derivative over $m$ and returning to the variable $r$, we obtain

$$
\frac{\partial \delta E}{\partial m}=-\frac{1}{m} \int d^{3} r \Psi^{+}(r) r \frac{\partial \delta V(r)}{\partial r} \Psi(r),
$$

and

$$
\delta g=-\frac{\kappa}{2 j(j+1)}\left[1+\frac{2 \kappa}{m}\left\langle r \frac{\partial \delta V(r)}{\partial r}\right\rangle\right] .
$$

Eqs. (9), (14) are the basis of our approach.

\section{ANOMALOUS MAGNETIC MOMENT}

It may be interesting to generalize our results to the case of a particle with a non-vanishing anomalous magnetic moment. In particular, it is necessary for antiprotonic atoms. The anomalous magnetic moment of the antiproton is big because of the complicated internal structure of the particle. Therefore, it makes sense to consider the modification of Eqs. (9), (14) due to the anomalous magnetic moment separately from the radiative corrections.

As known, the modified Dirac hamiltonian for a particle with the anomalous magnetic moment in an arbitrary electromagnetic field is of the form (see, e.g., [1])

$$
H=\gamma_{0}(\boldsymbol{\gamma} \cdot(\boldsymbol{p}+e \boldsymbol{A}))+\gamma_{0} m-e A_{0}+\frac{i \mu_{a}}{2} \gamma_{0} \sigma_{\mu \nu} F^{\mu \nu},
$$

where $\sigma_{\mu \nu}=(1 / 2)\left[\gamma_{\mu}, \gamma_{\nu}\right], \mu_{a}$ is the dimensional anomalous magnetic moment, $F^{\mu \nu}$ is the tensor of the electromagnetic field; we remind that the charge of the bound particle is $(-e)$.

As follows from Eq. (15), the correction to the energy of a bound particle in the homogenous magnetic field (2) is of the form (cf. Eq. (1))

$$
\Delta E=\int d^{3} r \bar{\Psi}(\boldsymbol{r})\left\{e(\boldsymbol{\gamma} \cdot \mathbf{A})-\mu_{a}(\boldsymbol{\Sigma} \cdot \mathbf{B})\right\} \Psi(\boldsymbol{r}),
$$

where $\Sigma_{j}=(i / 2) \epsilon_{j k l} \sigma_{k l}$ is the spin operator. The wave function $\Psi(\boldsymbol{r})$ is an eigenfunction of the hamiltonian (15) with $-e A_{0}(r)=V(r)$ and $A_{i}=0$. Since the potential $A_{0}(r)$ is spherically symmetric, the wave function still has the form (3), and the result for the $g$ factor reads (cf. Eq. (4))

$$
\begin{aligned}
g & =\frac{2 m}{j(j+1)} \times\left\{\kappa \int d r r^{3} f_{1} f_{2}\right. \\
& \left.-\frac{\mu_{a}}{2 e}\left[\int d r r^{2}\left(f_{1}^{2}-f_{2}^{2}\right)-2 \kappa\right]\right\} .
\end{aligned}
$$

The radial wave functions $f_{1}$ and $f_{2}$ satisfy the system of equations (cf. 1])

$$
\begin{aligned}
& f_{2}^{\prime}+(E-V-m) f_{1}+\left(\frac{1-\kappa}{r}-\frac{\mu_{a}}{e} V^{\prime}\right) f_{2}=0 \\
& f_{1}^{\prime}-(E-V+m) f_{2}+\left(\frac{1+\kappa}{r}+\frac{\mu_{a}}{e} V^{\prime}\right) f_{1}=0
\end{aligned}
$$


Then we multiply the first equation by $r^{3} f_{2}$ and the second one by $r^{3} f_{1}$, sum up the results and take the integral over $r$. Integrating by parts the terms with the derivatives, we arrive at the following relation (cf. (5))

$$
\begin{aligned}
\int d r r^{3} f_{1} f_{2} & =-\frac{1}{4 m}\left[1-2 \kappa \int d r r^{2}\left(f_{1}^{2}-f_{2}^{2}\right)\right. \\
& \left.-\frac{2 \mu_{a}}{e} \int d r r^{3} V^{\prime}\left(f_{1}^{2}-f_{2}^{2}\right)\right] .
\end{aligned}
$$

Using this formula and Eqs. (6) and (8), we present the result for the $g$ factor in the form

$$
\begin{aligned}
g & =\frac{1}{2 j(j+1)}\{-\kappa(1+2 a) \\
& +\left(2 \kappa^{2}+a\right) \frac{\partial E}{\partial m} \\
& \left.-a \frac{\kappa}{m} \int d r r^{3} V^{\prime}\left(f_{1}^{2}-f_{2}^{2}\right)\right\},
\end{aligned}
$$

where $a=-2 m \mu_{a} / e$ is the dimensionless anomalous magnetic moment (for a free particle $g=2(1+a)$ ). This result is obtained for an arbitrary potential $A_{0}$ and is exact in the parameter $a$. We emphasize that the radial wave functions $f_{1}$ and $f_{2}$ and the binding energy $E$ depend on the anomalous magnetic moment because of the equations (18). into

In the nonrelativistic approximation, Eq. (20) turns

$$
\begin{aligned}
g & =\frac{1}{2 j(j+1)}\{(1-2 \kappa)(a-\kappa) \\
& \left.-\left(2 \kappa^{2}+2 \kappa a+a\right) \frac{1}{2 m} \int d r r^{3} V^{\prime} f_{n r}^{2}\right\}
\end{aligned}
$$

where $f_{n r}$ is the radial part of the nonrelativistic wave function. This formula is valid even if $a \sim 1$.

It is interesting also to consider an expansion of the $g$ factor (20) in the parameter $a$ for arbitrary field strength (when the nonrelativistic approximation is not valid). The linear in this parameter term reads

$$
\begin{aligned}
\delta g_{a} & =\frac{a}{2 j(j+1)}\left\{-2 \kappa+\frac{\partial E}{\partial m}\right. \\
& -\frac{2 \kappa^{2}}{m} \frac{\partial}{\partial m} \int d r r^{2} V^{\prime} f_{1} f_{2} \\
& \left.-\frac{\kappa}{m} \int d r r^{3} V^{\prime}\left(f_{1}^{2}-f_{2}^{2}\right)\right\} .
\end{aligned}
$$

Here we used the formula for the linear in $\mu_{a}$ correction to the energy,

$$
\delta E_{a}=\frac{2 \mu_{a}}{e} \int d r r^{2} V^{\prime} f_{1} f_{2},
$$

and took into account that $\partial \mu_{a} / \partial m=0$. Let us consider Eq. (22) for the pure Coulomb potential. Strictly speaking, the equations (18) have no sense for a pure
Coulomb field because of the terms $\propto 1 / r^{2}$, which lead to the phenomenon of falling to the center. It is a consequence of the point-like source of the field and is absent if finite nuclear size is taken into account. However, for large quantum numbers which are mostly interesting for the experiments, the correction due to the finite nuclear size does not change essentially the result obtained from Eq.(22) for a pure Coulomb field. Using the radial matrix element from Ref. [4], we obtain

$$
\begin{aligned}
\delta g_{a} & =\frac{a}{2 j(j+1)}\left\{-2 \kappa+\frac{\gamma+n_{r}}{N}-\frac{\kappa(Z \alpha)^{2}}{N^{2}}\right. \\
& \left.-\frac{4 \kappa^{2}(Z \alpha)^{4}\left[2 \kappa\left(\gamma+n_{r}\right)-N\right]}{\gamma\left(4 \gamma^{2}-1\right) N^{4}}\right\},
\end{aligned}
$$

where $N$ and $\gamma$ are defined after Eq. (10). We see that the correction to the $g$ factor due to the anomalous magnetic moment has essentially more complicated dependence on quantum numbers than the leading term (10).

\section{FINITE-NUCLEAR-SIZE EFFECT}

As an illustration of efficiency of our method, let us consider a finite-nuclear-size correction to the boundelectron $\mathrm{g}$ factor. For $1 s$ state, this problem was solved analitically in Ref. [5] in the nonrelativistic approximation $(Z \alpha \ll 1)$, and numerically in Ref. [6] for arbitrary value of the parameter $Z \alpha$. In Ref.[7], the result was obtained analytically in the next-to-leading approximation in the parameter $Z \alpha$, and for arbitrary states. The result of Ref. 7] allows one to describe well the correction to the $g$ factor up to $Z=20$. On the other hand, in Ref. [8] the approximate formulas for the finite-nuclear-size correction to the energy levels, $\delta E_{f n s}$, were obtained, which gives the result with a relative error of about $0.2 \%$ up to $Z=100$. Using Eq. (9) and known dependence of $E_{f n s}$ on $m$, see [8], we obtain

$$
\delta g_{f n s}=\frac{\kappa^{2}}{j(j+1)} \frac{\partial E_{f n s}}{\partial m}=\frac{\kappa^{2}(2 \gamma+1)}{j(j+1)} \frac{E_{f n s}}{m} .
$$

Moreover, using our method we can calculate the radiative correction to $\delta g_{f n s}$ for $s_{1 / 2}$ state and $p_{1 / 2}$ state (and arbitrary $n_{r}$ ) coming from the effect of vacuum polarization. For these states, the finite-nuclear-size effect is the most significant. The radiative correction $\delta E_{f n s}$ to the energy $E_{f n s}$ was considered in detail in Ref. $[9]$. The correction due to the vacuum polarization $\delta E_{f n s}^{V P}$ can be represented in the form

$$
\delta E_{f n s}^{V P}=E_{f n s} \Delta^{V P},
$$

where $E_{f n s}$ was obtained in Ref. [8], and the explicit analytical form of $\Delta^{V P}$ for $s_{1 / 2}$ state and $p_{1 / 2}$ state is derived in Ref. 9]. Eq. [26) with $E_{f n s}$ from Ref. [8] and $\Delta^{V P}$ from Ref. [9] provides the high accuracy of $\delta E_{f n s}^{V P}$ up to $Z=100$. However, the correction $\Delta^{V P}$ was calculated in [9] for the mass of the bound particle $m$ being 
equal to the mass $M$ of the particle in the fermion loop. Therefore, in order to use Eq. (9) we should know the explicit dependence of $\Delta^{V P}$ on $m / M$ (we can set $M=m$ only after the differentiation over $m$ ). The corresponding expression for $\Delta^{V P}$ can be easily obtained following the derivation of $\Delta^{V P}$ in [9]. For $Z \alpha \sim 1$, the main contribution to $\Delta^{V P}$ is given by the logarithmically enhanced term

$$
\Delta^{V P} \approx \frac{2 \alpha(Z \alpha)^{2}}{3 \pi \gamma_{1}} \ln ^{2}(m R)
$$

where $\gamma_{1}=\sqrt{1-(Z \alpha)^{2}}$, and $R$ is the nuclear radius. This term is the same for $s_{1 / 2}$ and $p_{1 / 2}$ states and comes from the distances $1 / m, 1 / M \gg r \gg R$. Therefore, it contains only the logarithmic dependence on the mass of the bound particle, in contrast to the power-like dependence of $E_{f n s}$. Within the logarithmic accuracy, we have

$$
\frac{\partial}{\partial m} \delta E_{f n s}^{V P} \approx \Delta^{V P} \frac{\partial}{\partial m} E_{f n s}=\left(2 \gamma_{1}+1\right) \frac{\delta E_{f n s}^{V P}}{m} .
$$

Thus, for $Z \alpha \sim 1$, we obtain

$$
\delta g_{f n s}^{V P}=\frac{4}{3}\left(2 \gamma_{1}+1\right) \frac{\delta E_{f n s}^{V P}}{m} .
$$

For $Z \alpha \ll 1$ the dependence of $\Delta^{V P}$ on $m / M$ is more complicated and will be considered elsewhere.

\section{CONCLUSION}

We present a new effective approach to obtain various corrections to the bound particle $g$ factor using the corresponding corrections to the energy levels. It should be noted that this method is appropriate for the perturbations of potential-like type. There are also corrections which cannot be derived via this approach. The leading part of the non-potential contribution is due to the virtual Delbrück scattering and it was calculated in Ref. 10.

As other applications of our method, we can suggest the Uehling potential contribution to the $g$ factor of bound electrons and muons. In muonic atoms it is one of the dominant effects, while in electronic hydrogen-like atoms the accuracy is essentially higher (see, e.g., [11]).

For muonic atoms, the Uehling-potential correction to the energy levels was calculated in the nonrelativistic approximation for certain levels in 12]. The result exact in $Z \alpha$ was obtained in [13] for ground state of hydrogen-like atom and agrees with Eq. (9). The methods developed there can be easily applied to arbitrary levels and thus, using Eq. (9), one can find the correction to the $g$ factor due to the Uehling potential.

Using the known corrections to the energy levels coming from the high-order terms of vacuum polarization, we can obtain the corresponding contribution to the $\mathrm{g}$ factor. One correction comes from the Wichmann-Kroll potential (which accounts for the higher-order in $Z \alpha$ terms in the induced charge density), see Review [14]. Others are the second-order correction with respect to the Uehling potential, and first-order correction with respect to the radiative correction to the Uehling potential (of order of $\alpha)$.

\section{Acknowledgements}

We are grateful to V.G. Ivanov for useful discussions. A.I.M. thanks the Max-Planck-Institute for $\mathrm{Nu}$ clear physics, Heidelberg, and the Max-Planck-Institute for Quantum Optics, Garching, for hospitality during the visit. The work was supported in part by RFBR Grants No. 03-02-16510, 03-02-04029, and 03-02-16843, and DFG GZ: 436 Rus 113/769/0-1R.
[1] V. B. Berestetskii, E. M. Lifshitz, and L. P. Pitaevskii, Relativistic quantum theory (Pergamon Press, Oxford, 1982).

[2] M. E. Rose. Relativistic Electron Theory. John Wiley \& Sons, Inc., New York, London, 1961.

[3] S. A. Zapryagaev, Opt. Spect. 47, 9 (1979).

[4] V. M. Shabaev, in: Precision physics of simple atomic systems ed. by S. G. Karshenboim and V. B. Smirnov (Springer, Berlin, Heidelberg, 2003), p. 97.

[5] S. G. Karshenboim, Phys. Lett. A 266, 380 (2000).

[6] T.Beier, Phys. Rep. 339, 79 (2000).

[7] D. A. Glazov and V. M. Shabaev, Phys. Lett. A 297, 408 (2002).

[8] V. M. Shabaev, J. Phys. B: At. Mol. Opt. Phys. 26, 1103 (1993).

[9] A.I.Milstein, O.P.Sushkov, I.S.Terekhov, Phys. Rev. A 69, 022114 (2004).

[10] R. N. Lee, A. I. Milstein, I. S. Terekhov, and S. G.
Karshenboim, Phys. Rev. A 71, 052501 (2005).

[11] H. Häffner, T. Beier, N. Hermanspahn, H.-J. Kluge, W. Quint, S. Stahl, J. Verdú, and G. Werth, Phys. Rev. Lett. 85, 5308 (2000);

J. L. Verdú, S. Djekic, S. Stahl, T. Valenzuela, M. Vogel, G. Werth, T. Beier, H.-J. Kluge, and W. Quint, Phys. Rev. Lett. 92, 093002 (2004).

[12] G. E. Pustovalov, Sov. Phys. JETP 5, 1234 (1957);

D. D. Ivanenko and G. E. Pustovalov, Adv. Phys. Sci. 61, 1943 (1957)

[13] S. G. Karshenboim, Can. J. Phys. 76, 169 (1998); JETP 89, 850 (1999); misprints in some asymptotics were corrected in: S. G. Karshenboim, V. G. Ivanov and V. M. Shabaev, Can. J. Phys. 79, 81 (2001); JETP 93, 477 (2001).

[14] E. Borie and G.A. Rinker, Rev. Mod. Phys. 54 , 67 (1982). 